NASA TECHNIEAC MEMOREND

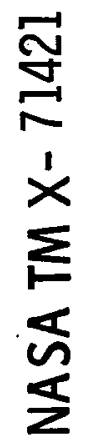

PLASMA FLUCTUATIONS IN A KAUFMAN THRUSTER

by John S. Serafini and Fred F. Terdan

Lewis Research Center

Cleveland, Ohio 44135

TECHNICAL PAPER proposed for presentation at Tenth Electric Propulsion Conference sponsored

1 by the American Institute of Aeronautics and Astronautics Lake Tahoe, Nevada, October 31 - November 2, 1973 


\section{PLASMA FLUCTUATIONS IN A KAUFMAN THRUSTER}

John S. Serafini and Fred F. Terdan

National Aeronautics and Space Administration

Lewis Research Center

Cleveland, Ohio

\section{Abstract}

Measurements of the RMS magnitude, spectra and cross-correlations for the fluctuations in the beam, discharge and neutralizer keeper currents are presented for a 30-cm diameter dished grid ion thruster for a range of magnetic baffle currents and up to 2.0 amperes beam current. The ratio of RMS to mean ion beam current varied from 0.04 to 0.23 . The spectra of the amplitudes of the beam and discharge current fluctuations were taken up to $9 \mathrm{MHz}$ and show that the predominant amplitudes occur at frequencies of $10 \mathrm{kHz}$ or below. The falloff with increasing frequency is rapid. Frequencies above $100 \mathrm{kHz}$ the spectral levels are $45 \mathrm{~kb}$ or more below the maximum peak amplitudes. The crosscorrelations revealed the ion beam fluctuations to have large radial and axial scales which implied that the beam fluctuates as a whole or "in-phase". The cross-correlations of the beam and neutralizer keeper current fluctuations indicated the neutralizer contributions to the beam fluctuations to be small, but not negligible. The mode of operation of the thruster (values of beam and magnetic baffle currents) was significant in determining the RMS magnitude and spectral shape of the beam fluctuations. The major oscillations were not found to be directly dependent on the power conditioner inverter frequencies.

\section{Introduction}

The 30-cm diameter ion thruster is being considered for prime propulsion for solar electric propulsion missions. $(1,2,3)$ In these missions electromagnetic noise, or fluctuations that might be produced by thruster operation is of possible concern to the areas of communication, science, and overall system operation. $(4,5)$ In addition, information on fluctuations can possibly aid in optimization of the thruster subsystem.

In the investigation presented herein, initial measurements were obtained of the fluctuations of the ion beam current at a number of points in the beam over range of thruster operating conditions. Several aspects of these fluctuations were recorded $(6,7,8,9)$ which included 1) the overall magnitude or intensity given as the RMS (root-meansquare), 2) the frequency spectral distribution, and 3) both auto-correlations and space-time correlations (i.e., cross-correlations) of the fluctuations at two points in the beam. Cross-correlations of the beam current fluctuations with the discharge and neutralizer current fluctuations were also obtained.

A $30-\mathrm{cm}$ diameter thruster $(10,11,12,13)$ fitted with dished grids, was used in this study. The power conditioner (14) used was a high-frequency transistor inverter type.

Apparatus and Experimental Technique

\section{Thruster and Power Conditioner}

The 30-cm hollow cathode thruster used in this investigation is described in reference 10. The thruster was provided with a set of high-perveance compensated dished grid optics similar to set $M$ in reference 11 . Slight modifications were also made to the discharge chamber geometry (identical to thruster E-2 in reference 11). The thruster thus incorporates a magnetic baffle circuit with four turns that required a constant-current power supply separate from the power conditioner for the thrus-
ter. $(12,13)$

The power conditioning console was initially designed and fabricated by Hughes Research Laboratories under Government contract (10) The system block diagram is shown in Fig. 1. The screen supply was constructed by series connecting the DC outputs of $8 \mathrm{high}$ frequency $(10 \mathrm{kHz})$ inverters. The discharge supply was configured with 3 series connected inverters and the accelerator supply was preceded by a closed-loop line regulator. Keeper, heater, and vaporizer power was supplied by a $5 \mathrm{KHz}$ inverter. $\mathrm{All}$ outputs except the positive and negative high voltages were constant current controlled with a magnetic-amplifier.

The only major modifications made to this unit were in the area of control $(14)$. These changes were added to insure stable operation and high efficiency thruster operation using high perveance ion extraction systems.

\section{Thruster Operation}

The thruster was mounted as shown in Fig. 2 and was inserted through a $92-\mathrm{cm}$ diameter port in a 7.6 meter diameter by 18.3 meter long vacuum chamber maintained at a nominal tank pressure of $10^{-7}$ torr. The thruster was operated at beam currents, $J_{B}$ (see Appendix A for a complete list of symbols) of $0.85,1.45$ and 2.0 amperes. The screen $\left(V_{I}\right)$ and accelerator $\left(V_{A}\right)$ grid voltages were 1100 and -600 volts, respectively. Unless otherwise specified, the energy expenditure per beam ion was kept constant as $187 \mathrm{ev} / \mathrm{ion}$ with the discharge voltage, $\Delta \mathrm{V}_{I}$, constant at 37.4 volts. The current to the magnetic baffle was varied from 0 to 13.0 amperes depending on the value of $\mathrm{J}_{\mathrm{B}}$. For these conditions the discharge utilization varied from 0.85 to 0.95 . For all of the data presented herein the beam current and discharge voltage were closed loop controlled.

Transducers

Two current probes were used to sense ion beam current fluctuations (see Figs, 2 and 3 ). The probes could be translated axially from $2.5 \mathrm{~cm}$ to $64.0 \mathrm{~cm}$ relative to the center of the dished accelerator grid. Rotation of the probes about their 
support-arms allowed measurements to be made at up to $56 \mathrm{~cm}$ radially from the centerline of the thruster.

The ion beam probe leads were electrically shielded, thermally insulated, and fed through the support-arm into a vacuum feed-through. For both probes a shielded variable-voltage bias supply was used which permitted a variable polarity bias with respect to facility ground to be applied. For most of the data the bias was -20 volts. The net ion or electron current to the probe was passed through a $100 \Omega$ resistor connected to ground. The voltage drop across the resistor was then used directly to measure current. The time-mean voltages were monitored on DC digital voltmeters and the time-varying voltages were fed into cathode followers having an input impedance of $400 \mathrm{M} \Omega$ shunted by $4 \mathrm{pf}$. An AC coupled amplifier provided a suitable output of the time-varying signal. It was possible to inspect portions of the frequency content of the broad-band data by the use of high-pass band or low-pass band frequency filtering.

Two current loop probes were used to sense emission current and neutralizer keeper current fluctuations. These probes were placed about the appropriate current-carrying cables from the power conditioner to the thruster. The sensitivity of the loops measuring the emission current and neutralizer keeper current was 4 amps per volt and 10 milliamps per millivolt, respectively.

\section{Analysis of Time-Varying Data}

The magnitudes of the time-varying output signals from the ion beam probe and current loopprobe systems were measured on meters which gave the true root-mean-square magnitude. This RMS measurement was made for all of the experimental tests performed. For these signals the amplitude as a function of frequency was measured on three heterodyne-type spectrum analyzers which permitted an examination of the frequency spectral distribution to about $10 \mathrm{MHz}$. For most of the experimental tests the spectra were monitored over ranges such as 0 to $1 \mathrm{MHz}, 0$ to $100 \mathrm{kHz}, 0$ to $20 \mathrm{kHz}$ and 0 to $10 \mathrm{kHz}$. In addition, several tests were made in which the output signals were subjected to analysis by high-pass band frequency filtering for a range of cut-off frequencies from $20 \mathrm{~Hz}$ to $2.0 \mathrm{MHz}$.

The final type of processing of the timevarying output signals was measuring space-time or cross-correlations as well as the auto-correlations of fluctuations in the beam. A brief description of this technique and its implications are given in Appendix $B$. Also measurements of the cross and auto correlation between fluctuations of either the discharge current or the neutralizer keeper current and the current fluctuations sensed by one of the ion beam probes were obtained.

\section{Calibration of the Data Systems}

The overall gain of the two ion beam probe systems was measured before, during, and after the program of investigation. Also, the amplitude response as a function of frequency was measured. The results of this calibration indicated that for both probe systems this response was constant to within $\pm 0.85 \mathrm{db}$ from $100 \mathrm{~Hz}$ to $6 \mathrm{MHz}$ to $10 \mathrm{MHz}$. For the same input signal the phase difference (of importance in the correlation studies as described in Appendix B) between the output signals for the two probe systems was negligibly small over the frequency range of interest ( $1 \mathrm{MHz}$ or less).

The overall gain and the amplitude response as a function of frequency was also determined for the discharge current loop and was constant to within $\pm 0.5 \mathrm{db}$ from $800 \mathrm{~Hz}$ to at least $1 \mathrm{MHz}$. For the same input signal the difference in phase of the output signal from the discharge current loop compared with the output signal from one ion probe beam system was less than $10^{\circ}$ up to $100 \mathrm{kHz}$ and less than $14^{\circ}$ up to $300 \mathrm{kHz}$ (maximum frequency range of the correlator). The overall gain and amplitude response as a function of frequency for the neutralizer keeper current loop was constant over the desired frequency range and the $3 \mathrm{db}$ down point at lower frequency end was $120 \mathrm{~Hz}$.

The true RMS meters used for the data and the calibration tests had a range of response to 10 MHz. For calibration purposes the input and output meters were comparison calibrated over this frequency range. For the spectrum analyzers the amplitude and frequency calibrations were made and used in calculating the data. The response and the operational behavior of the correlator was checked by using sine wave inputs and found to be constant to at least $250 \mathrm{kHz}$.

\section{Results and Discussion}

This section will present the results on RMS magnitude, spectral data, effect of ion beam probe bias, and the correlations in that order.

\section{RMS Magnitudes}

Figure 4 shows the radial variation of the time-mean and root-mean-square magnitude of the ion beam probe current fluctuations sensed at a fixed axial distance from the grid. This is given for a beam current, $J_{B}$, of 1.45 amps and magnetic baffle current, $J_{M B}$, of 6.0 amps. In this and all other figures, the functional dependence on time, $t$, will be omitted in the notation and thus

$\overline{I(t)} \rightarrow \bar{I}$ and $\sqrt{i^{2}(t)} \rightarrow \sqrt{i^{2}}$. From Fig. 4 it is seen that the trend of the RMS values of the fluctuations and the mean current are generally similar. Figure 5 presents the axial variation of time-mean and RMS magnitude probe currents of the fluctuations for $r=0$ for the same conditions as the previous figure.

Figure 6 shows the ratio. of the RMS magnitude to the time-mean currents of the ion beam probe fluctuations as a function of $J_{M B}$, the magnetic baffle current for a $J_{B}$ of 1.45 emps and 2.00 amps. Both curves in Fig. 6 exhibit similar, striking characteristics. As the value of $J_{M B}$ was increased the intensity of the fluctuations was first low (about 0.04). There was then a sharp rise over a small region of $J_{M B}$ to a new higher intensity level as $J_{M B}$ was further increased. For the $J_{B}=2.0$ amp data this phenomenon occurred at two narrow ranges of $J_{M R}$. Apparently, a mode transition occurred, possibly associated with a mode change in the discharge voltage-current characteristic as a function of cathode propellant flow-rate. 
A corresponding behavior in the fluctuations of the discharge current was observed. These results were obtained for $J_{B}$ of 1.45 amps and are given in Fig. 7. Not surprisingly, the discharge current fluctuations had a very similar behavior to the ion beam probe fluctuations as a function of $J_{M B}$. This plausibly suggests that the levels of fluctuations noted in the beam were primarily a consequence of the behavior of the plasma in the discharge chamber. More will be said on this point after the correlation results are presented.

Figure 8 presents the intensity as a function of $J_{B}$ for several values of $J_{M B}$. For constant $J_{M B}$ the intensity appeared to decrease as $J_{B}$ was increased. It is evident, however, that the dominant variable in determining the intensity was the magnetic baffle current.

Inasmuch as it was found that the discharge chamber conditions were significant in determining the intensity level of the beam fluctuations, it was of interest to determine the effect of varying $\Delta V_{I}$. Figure 9 shows the variation of the intensity of the ion $b$ m probe fluctuations as a function of $\Delta V_{I}$, for $J_{B}$ of 1.45 amps and a $J_{M B}$ of 0 and $6 . \overline{2}$ amps. For both values of $J_{M B}$ the variation was monotonic over the range of $\Delta V_{I}$ tested. The level of the fluctuation intensity was much more dependent upon the value of $J_{M B}$ than the discharge voltage. The curves in Figs. 7,8 , and 9 suggest that the fluctuations occurring in the beam originate as phenomena at the hollow cathode region of the discharge chamber.

Spectral Data

Figures 10,11 , and 12 are typical spectra which show the relative amplitude as a function of frequency of the fluctuations in ion beam probe current, the discharge current, and the neutralizer keeper current, respectively, for a beam current of 2.0 amps and $a J_{M B}$ of 13.0 amps. These spectra are typical in spectral shape but as will be discussed later the frequencies of the dominant peaks do vary. In Fig. 10 the largest amplitudes are seen to be at frequencies below $10 \mathrm{kHz}$. There are several peaks in this region (the frequencies and magnitudes of which are a function of the thruster operating parameters). At frequencies higher than this peak-region, a rapid fall-off in amplitude with frequency occurs. This is generally characteristic of all the spectral data for all thruster operating parameters used in this investigation. The sharp drop in amplitude is about 45 $\mathrm{db} /$ decade which is equivalent to a $f^{-4.5}$ dependence for the spectral amplitude. This is a more negative slope than the $f^{-2.5}$ dependence found in the spectra of plasma turbulence $(15,16,17)$ There are several possible reasons for this behavior. For example, the neutralized beam plasma consisting of ions with directed energy is quite different physically from the plasmas of the earlier works. Another possibility, the power conditioner may interact with the thruster in a special manner.

A final point to note about Fig. 10 is the behavior for frequencies greater than $100 \mathrm{kHz}$. Here is seen a rise in amplitude of about 5 or $6 \mathrm{db}$ to peak at about $700 \mathrm{kHz}$ and a sharp drop-off near $3 \mathrm{MHz}$ with a peak at about $7.5 \mathrm{MHz}$. However, the amplitudes at these higher frequencies (above 100 $\mathrm{kHz}$ ) are more than $45 \mathrm{db}$ below the maximum peak amplitudes (i.e., the amplitudes at the se higher frequencies are less than $10^{-2}$ of those at frequencies less than $10 \mathrm{kHz}$ ).

Many measurements of the spectra of the discharge current fluctuations, as in Fig. 11, were taken throughout the program. This was done to attempt to ascertain if the fluctuations in the beam were primarily a function of the discharge chamber fluctuations. Similar to the data of Fig. 10 the data of Fig. 11 exhibits several dominant peaks at frequencies near $10 \mathrm{kHz}$ and below. These peaks, however, did not occur at the same frequencies. This was generally found to be the situation for the other operating conditions as well. However, the fall-off in amplitude at frequencies higher than about $10 \mathrm{kHz}$ is again about $45 \mathrm{db} /$ decade and it continues to fall-off without any reversal in slope as in Fig. 10. Also evident in Fig. 11 as well as Fig. 10 are narrow peaks of small magnitude between 50 and $100 \mathrm{kHz}$. These are probably harmonics of the power supply inverter frequency $(10)$.

The relative amplitude of the neutrolizer keeper current fluctuations, in $\mathrm{db}$, is shown in Fig. 12 as a function of frequency. The RMS magnitude, $\sqrt{\overline{j_{n k}^{2}}}$, was found to be essentially constant at 0.29 . amps for all the operating conditions. The time-mean neutralizer keeper current, $\mathrm{J}_{\mathrm{nk}}$ was also constant at 1.96 amps. The intensity of the neutralizer keeper current fluctuations was, therefore, 0.148. The spectral data in Fig. 12 is somewhat different from the two previous figures. The raw data from the spectrum analyzers exhibited very sharp peaks (more than $40 \mathrm{db}$ in magnitude) which occurred at the fundamental and harmonic frequencies of the power conditioner inverter supplying the neutralizer keeper. The curve in Fig. 12 is the envelope of the maxima of these harmonic peaks. Also given in the figure are the locations of the fundamental and several of the observed harmonics. The harmonics with the highest peaks were the fifth and seventh at 27 and $38 \mathrm{kHz}$, respectively. At lower and higher frequencies there is a fall-off in amplitude. Between $100 \mathrm{kHz}$ and $1 \mathrm{MHz}$ this fall-off appears to be roughly $20 \mathrm{db} /$ decade or a much smaller rate of fall-off for the $J_{n k}$ fluctuations than for the beam current fluctuations. There is a peak in Fig. 12 at about $600 \mathrm{kHz}$ which suggests that the behavior of the spectra of the beam fluctuations (Fig. 10) for frequencies about this point are related to the neutralizer-beam coupling phenomenon(18).

Figures 13 and 14 are given to illustrate further the importance of the lower-frequency regime in contributing to the RMS magnitude and also to show the effect of varying $\mathrm{J}_{\mathrm{MB}}$, the magnetic baffle current. In Fig. 13 for the ion beam probe fluctuations, the ratio

is given as a function of $f_{\mathrm{HP}}$

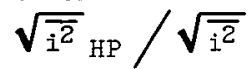

$$
\sqrt{\overline{i^{2}}}=\sqrt{\int_{0}^{\infty} \overline{i^{2}}(f) d f}
$$

and

$$
\left(\sqrt{\overline{i^{2}}}\right)_{\mathrm{HP}} \approx \sqrt{\int_{f_{\mathrm{HP}}}^{\infty} \overline{i^{2}}(f) d f}
$$


and $f_{H P}$ is the cut-off frequency for the highpass band of the frequency filter. This alternative manner of displaying the spectral characteristics of the ion beam probe fluctuations permits the estimation of the contribution of the frequency spectrum below the cut-off frequency $f_{H P}$ to the RMS magnitude of the fluctuations. It is readily seen, that as the magnetic baffle current is increased from 0 to 9.0 amps for a beam current of 1.45 anps, that the lower-frequency portion of the spectrum contributes more heavily to the RMS magnitude. For a $J_{M B}$ of 6.0 and $9.0 \mathrm{amps}$ the two curves are almost identical with the ratio dropping to 0.3 as $f_{\mathrm{HP}}$ increases to $10 \mathrm{kHz}$ and further dropping to 0.06 as $f_{\mathrm{HP}}$ increases to $1 \mathrm{MHz}$.

Figure 14 shows this type of plot for the discharge current fluctuations. The behavior is generally the same as for the ion beam probe fluctuations. The contribution at lower frequencies is even stronger here. For a $J_{M B}$ of 6.0 and 9.0 amps the two curves are practically identical with the ratio dropping to 0.52 as $f_{\mathrm{HP}}$ increases to $10 \mathrm{kHz}$ and further dropping to 0.01 as $f_{\mathrm{HP}}$ increases to $200 \mathrm{kHz}$.

Figure 15 shows the variation of the peakfrequencies of the spectra of the ion beam fluctuations as a function of $J_{M B}$, the magnetic baffle current for a beam current of 1.45 amps and $2.0 \mathrm{~V}$ amps. Show in the figure are data representing the "strongest peaks" and "next strongest peaks" of the spectra. This manner of display resulted from the occurrence of multiple peaks in the lowerfrequency regime for many of the operating conditions. It should be evident from Fig. 15 that the two sets of peak-frequencies are not fundamental and first harmonic frequencies. Although peaks at the harmonic frequencies do indeed occur in some instances, they are not considered in this discussion. The main point of the data in Fig. 15 is that for most of the data the peak-frequencies are not equal to the fundamental frequency of the inverters (about $5.4 \mathrm{kHz}$ ). Only for a $J_{B}$ of 2.0 amps and increasing $J_{M B}$ to 12 and 13 amps did the peak-frequency approach $5.4 \mathrm{kHz}$.

In Fig. 15 for $J_{B}$ of 1.45 amps at $J_{M B}=$ 0 amps the "strongest" peak-frequency is $1.4 \mathrm{kHz}$ and, at all other values of $J_{\mathrm{MB}}$, they are near $6 \mathrm{kHz}$ or above. In addition the "next strongest peaks" at a $J_{B}$ of 1.45 amps are also around $2 \mathrm{kHz}$ (as well as for $J_{B}$ of 2.0 amps). Thus, for both beam currents in Fig. 15, the frequencies of the "strongest" and "next strongest" peaks were not harmonics of each other. The two modes did not vary with $J_{M B}$ in the same way. This behavior indicates that dual modes of oscillation coexist. The frequencies of these modes appear to be independent of the inverter frequency and were functions of discharge plasma phenomena. Similar lowfrequency resonance behayior of discharge plasmas has been observed before $(19,20)$.

\section{Effect of Probe Bias Voltage}

Figure 16 presents the variation of the timemean and RMS magnitude of the beam probe current fluctuations as a function of the bias voltage on the ion beam probe. The variation of the probe bias voltage was initially done to check out the operation of the beam probe system. In addition, this procedure is equivalent to using these probes as Iangmuir probes and thus could yield some insight into the effect of the neutralizer electrons in producing fluctuations. It is seen from the figure that both the time-mean and RMS values of the fluctuations are virtually invariant with probe bias voltage from a bias voltage of -30 to nearly 0 volts. This is usually referred to as the "ionsaturgtion" portion of a Langmuir probe characteristic $(6)$. Because the beam plasma consists of streaming ions of considerably higher directed energy than is usually experienced in most plasmas (other than of the fusion-type), the ion current is not small as is usual. Otherwise, as the bias voltage is further increased the time-mean current rapidly becomes negative (collecting electrons) and the RMS magnitude of the current fluctuations rapidly increases.

In Fig. 17 the relative amplitude in $\mathrm{db}$ of the beam probe current fluctuations as a function of frequency is given for bias voltages, $V_{\text {bias }}$, of $-20,0$ and +3 volts. From the previous figure it can be seen that a $V_{b j}$ of -20 volts corresponds to a point on the ion-saturation" portion of the probe characteristic and +3.0 volts corresponds to a point on the "electron-collecting" portion of the probe-characteristic. There are two striking characteristics of the data in Fig. 17. One is that the amplitude level is significantly higher when it is "collecting electrons" and this was expected. The appearance of the very sharp, multiple, and large-amplitude peaks at frequencies higher than $10 \mathrm{kHz}$ was unexpected. By superimposing the spectrum of the neutralizer keeper current fluctuations on the spectra at $V_{\text {bias }}=+3.00$ volts, it was easily observed that the frequencies of the beam probe spectral peaks above $10 \mathrm{kHz}$ precisely agreed with those of the neutralizer keeper current spectra. Consequently, it is readily apparent that these peaks above $10 \mathrm{kHz}$ are simply evidence that the neutralizer electrons are excited by the harmonic frequencies of the inverter. Thus, positive bias of the probe provides a sensitive means of examining the low-level contributions of the neutralizer electron oscillations to the beam fluctuations. Further, it should be noted that the major contribution occurs at high frequencies (> $10 \mathrm{kHz}$ ) where the amplitudes of the fluctuations are substantially reduced.

\section{Correlations of the Fluctuations}

In order to determine the geometric size of the beam that is fluctuating as a whole ("inphase"), cross-correlations or space-time correlations were measured (see Appendix B). As indicated in Appendix B, the correlation curves were obtained in terms of $R$, the correlation coefficient, as a function of $\tau$, the time delay between the fluctuations measured at two points in the beam. The cross-correlation curves had a maximum, $R_{\max }$ usually near $\tau=0$ which implied that (for the given probe separation distances and time-resolvability of the correlation analyzer) the fluctuations did not have measurable convective velocities. (2I)

Figures 18 and 19 give the variation of $R_{\max }$ of the correlations of the beam fluctuations as $a$ function of radial and axial probe separation distances, respectively, for $J_{B}=1.45$ amps and $J_{M B}=6.0$ amps. For Fig. 18 both beam probes were at $\mathrm{Z}=17.8 \mathrm{~cm}$. One remained at $r=0$ and the other probe position varied across the radius. From Fig. 18 it is seen that the values of $R_{\max }$ 
are quite large (i.e., $\geq 0.65$ ) throughout the entire radial extent of the beam. This signifies that the fluctuations are "in phase" over the radial extent of the beam and that the radial scale of the fluctuations is as large as the beam diameter.

For Fig. 19 both beam probes were at $z=r=$ $4.8 \mathrm{~cm}$ and one was kept at $\mathrm{z}=17.8 \mathrm{~cm}$ and the other probe varied along the axis. From Fig. 19 it is seen that the values of $R_{\text {max }}$ are also quite large (i.e., >0.77) throughout the entire axial extent measured. This also signifies that over this axial region the beam fluctuations are "in phase" and, thus, the axial scale of the. fluctuations is greater than the maximum probe separation distance, $46.5 \mathrm{~cm}$. An interesting aspect of the data shown in Fig. 19 was the trend for the values of $R_{\max }$ to increase as the axial probe separation distance increased. There are several possible explanations for this behavior. The most plausible is that there was a vefy rapid convective aspect of the fluctuations (21) along the beam axis and that as the two probes were further separated axially, the $R_{\max }$ then should be expected to increase up to a certain distance (and then decrease for greater axial separation distance).

Another possible explanation is that the upstream probe could have produced a disturbance which propagates toward the downstream probe and thus would be able to effect the fluctuations sensed by the downstream probe. At the time of the tests this possibility was checked out. This was done by measuring the output of the downstream probe with and without the upstream probe in place. The results indicated that there was no disturbance effect on the second or downstream probe. The results in the two figures thus indicate that the entire beam (at least the ions) fluctuated "in phase", or as a whole.

Figure 20 gives the variation of $R_{\max }$ of the beam fluctuations as a function of $J_{B}$ for various values of $J_{M B}$. For the entire figure the probe separation distances were constant, $\zeta_{r}=4.8 \mathrm{~cm}$ and $\zeta_{z}=0$. The results of the figure indicate an increasing trend in $R_{\max }$ as $\mathrm{J}_{\mathrm{B}}$ increased. The $R_{\max }$ is only down to 0.70 at a $J_{B}$ of 0.85 amps and the scale or size of the fluctuations is large even at a $J_{B}$ of 0.85 amps.

When it was observed that the beam fluctuations were not small in intensity and scale it was decided to investigate the possible relationship of the discharge current fluctuations with the beam fluctuations. This was done by measuring the cross-correlations between these fluctuations and the beam fluctuations. Figure 21 gives the variation of $R_{\max }$ of the correlations of the beam and discharge current fluctuations as a function of the beam current, $J_{B}$, for various values of $J_{M B}$. For the entire figure the beam probe was at $\mathbf{x}=0$ and $z=17.8 \mathrm{~cm}$. For all the data in the figure the values of $R_{\max }$ are large and the effect of $J_{M B}$ seems to be not greatly significant. However, as $J_{B}$ increased from 0.85 to 2.00 amps the $R_{\max }$ decreased from about 0.81 to about 0.62 . This can possibly be explained by suggesting that as $J_{B}$ increased the beam fluctuations did in fact become less dependent on the fluctuations of the discharge current. Not only were the values of $R_{\text {ax large }}$ for the cross-correlations of the beam and dis- charge current fluctuations, but also it was found that the $R_{\max }$ did not occur at or near $\tau=0$ (this is not presented here). This is direct evidence that there existed a convective relationship between the discharge and beam current fluctuations.

Figure 22 gives the variation of $R_{\max }$ of the correlations of the beam and neutralizer keeper current fluctuations as a function of the beam current, $J_{B}$. While the values of $R_{\text {max }}$ are much smaller than the values for the cross-correlations of the beam and discharge current fluctuations, they are not insignificantly small. This result indicates that the neutralizer fluctuations did in some manner influence the beam fluctuations. Also, the results in Fig. 22 indicate that there was no significant effect of varying $J_{B}$ on the values of $R_{\max }$ and thus the effect of the neutralizer keeper current fluctuations on the beam current fluctuations was not greatly sensitive to $J_{B}$.

\section{Concluding Remarks}

An experimental investigation of the fluctuations in beam current of a 30-cm electron-bombardment (Kaufman) thruster with a switching transistor powex conditioner has been performed. Studied were a range of beam currents, $J_{B}$, of 0.85 to 2.00 amps and a range of magnetic baffle currents, $J \mathrm{MB}$, of 0 to 13.0 amps at an energy expenditure of 187 ev/ion and discharge voltage of 37.4 volts for most of the data. The results and concluding remarks include the following:

1) The beam current fluctuation intensity defined as the ratio of the RMS magnitude of the fluctuating to time-mean currents varied from 0.04 to 0.23 .

2) Spectra of the amplitudes of the beamcurrent fluctuations as a function of frequency to $9 \mathrm{MHz}$ show that the predominant amplitudes occur at frequencies of $10 \mathrm{kHz}$ or below. The fall-off with increasing frequency is rapid such that at frequencies above $100 \mathrm{kHz}$ the spectral levels are $45 \mathrm{db}$ or more below the maximum peak amplitudes occurring at frequencies below $10 \mathrm{kHz}$.

3) The cross-correlations of the beam fluctuations indicate that over the radial and axial extents, the beam fluctuates as a whole or "in phase".

4) As observed from the spectral and crosscorrelation results, the discharge current fluctuations directly contribute to the beam-current fluctuations.

5) The RMS magnitudes, spectra and crosscorrelation measurements of the neutralizer keeper current fluctuations indicate that the neutralizer contributions to the beam fluctuations were small but not negligible and appear mostly in the higher frequency range measured.

6) The mode of operation of the thruster (values of the beam current, $J_{B}$, and the magnetic baffle current, $J_{M B}$ ) are significant in determining the RMS magnitude and spectral shape of the beam fluctuations. 
7) The apparent sources of the fluctuations were found to be:

a) coherent oscillations in the discharge chamber plasma, and

b) to a lesser extent coupling phenomens between the neutralizer electrons and the beam.

8) The intensity of the fluctuations decreased as the beam current was increased (for a constant magnetic baffle current).

\section{Appendix A}

List of Symbols

f frequency, $\mathrm{Hz}$

$f_{\mathrm{HP}} \quad$ high-pass cut-off frequency, $\mathrm{Hz}$

$I(t)=\overline{I(t)}+i(t) \quad$ sum of time-mean and timevarying beam probe current, amps

$i(t) \quad$ time-varying probe current, amps

$J_{B} \quad$ beam current, amps

$J_{e}(t)=\overline{J_{e}(t)}+j_{e}(t) \quad$ sum of time-mean and timevarying discharge (emission) currents, amps

$j_{e}(t)$ time-varying discharge (emission) current, amps

$\mathrm{J}_{\mathrm{mb}} \quad$ magnetic baffle current, amps

$J_{n k}(t)=\overline{J_{n k}(t)}+j_{n k}(t)$ sum of time-mean and time-varying neutralizer keeper currents, amps.

$j_{n k}(t)$ time-varying neutralizer keeper current, amps

$R^{\prime}(\tau)$ auto- or cross-correlation, amps ${ }^{2}$ (see Appendix B)

$R(t)$ auto- or cross-correlation coefficient, dimensionless (see Appendix B)

$R_{\max }$ maximum correlation coefficient at a given probe separation

$r \quad$ radial probe distance from grid-center, $\mathrm{cm}$

Vias probe bias, volts

$\mathrm{V}_{\mathrm{I}} \quad$ screen grid potential, volts

$\mathrm{V}_{\mathrm{A}} \quad$ accelerator grid potential, volts

$\Delta V_{I} \quad$ discharge voltage, volts

z axial probe distance from grid-center, cm

$\tau \quad$ time-delay in correlation analysis, msecs (see Appendix B)

$\zeta_{r}$ radial separation distance between two probes, cm

$\zeta_{\mathrm{z}} \quad$ axial separation distance between two probes, cm
Appendix B

Analysis of Time-Varying Data

A physical quantity, $E(t)$, may be defined as

$$
E(t)=\bar{E}+e(t)
$$

where

$$
E \equiv \frac{1}{T} \int_{0}^{T} E(t) d t
$$

and $T$ is any suitable integration time.

$\bar{E}$ is then the time-mean of $E(t)$.

The time-mean of $e(t)$ is, therefore, by definition

$$
\overline{e(t)} \equiv 0
$$

of course, it is precisely the characteristics of this quantity, $e(t)$, that is of interest in describing a possible "noise" source. First the magnitude of the fluctuation is considered. Thus,

$$
\overline{e^{2}(t)}=\frac{1}{T} \int_{0}^{T} e^{2}(t) d t
$$

(this is not zero as long as there are any fluctuations: about the time-mean value) It is also possible to consider $\overline{e^{2}(t)}$ in terms of its frequency spectral components as

$$
\overline{e^{2}(t)}=\int_{0}^{\infty} F(f) d f
$$

where $F(f)$ is the spectral density. Now the auto-correlation function of $e(t)$, is defined as

$$
R^{\prime}(\tau)=\overline{e(t) e(t+\tau)}=\frac{T}{T} \int_{0}^{T} e(t) e(t+\tau) d t
$$

where $\tau$ is a time-delay variable. If $\tau=0$, then $R^{\prime}(0)=e^{2}(t)$, the mean-square of the fluctuations. Also, the auto-correlation function, $R(\tau)$, is the Fourier transform of the spectral density, $F(f)$, as

$$
R^{\prime}(\tau)=\text { (const) } \int_{0}^{\infty} F(w) e^{-i w \tau} d w
$$

where $\omega=\frac{f}{2 \pi}$.

The information presented so far on the fluctuating quantity, $e(t)$, involves only the measurement at a single point of a single parameter. It gives no information on how much of the region probed is fluctuating as a whole of "inphase". Also, it cannot give information as to whether or not the fluctuating region possesses convective or rotating velocities. In order to obtain this kind of information, one must consider the cross-correlation function of the fluctuations, $e_{1}(t)$ and $e_{2}(t)$, measured by two probes at a separation distance $\zeta$. Thus,

$$
\begin{array}{r}
R^{\prime}(x, \zeta, \tau)=\overline{e_{1}(x, t) e_{2}(x+\zeta, t+\tau)} \\
=\frac{1}{T} \int_{0}^{T} e_{1}(x, t) e_{2}(x+\zeta, t+\tau) d t
\end{array}
$$


It is usually desirable when correlation functions are considered to have them in a dimensionless format. Convention (and logic) dictates that for the auto-correlation function

$$
R(\tau)=\frac{R^{\prime}(\tau)}{e^{2}(t)}
$$

and for the cross-correlation function

$$
R(\dot{x}, \zeta, \tau)=\frac{R^{\prime}(x, \zeta, \tau)}{\sqrt{\overline{e_{1}^{2}(t)}} \sqrt{\overline{e_{2}^{2}(t)}}}
$$

The correlation functions are presented in their dimensionless form in the present paper. The correlator used in the present study performs the computation of the auto- or cross-correlation functions in essentially the dimensional form and as a function of $\tau$, the time delay.

Examination of $R(x, \zeta, \tau)$ as functions of $\zeta$ and $\tau$ gives the space-time correlations of the fluctuations. Much information on the fluctuations can be gained from such an analysis. For the present purposes it is sufficient to just point out several salient features. If the cross-correlation coefficient is zero everywhere for all $\tau$ and $\zeta$, then the fluctuations at points 1 and 2 are not related to each other or "in-phase". This is also true for the case where one of the fluctuating signals is from the ion beam current probe and the other is from one of the current loops. On the other hand if the correlation coefficient is, say, $0.5<R \leqq 1.0$, then the two signals $e_{l}(t)$ and $e_{2}(t)$ are said to be highly correlated or nearly "in phase" with each other. If the correlation equals 1, then they are exactly "in phase". Thus, one can choose to specify (somewhat arbitrarily) that separation distance, $\zeta$, over which $R \geqq 0.5$ as the scale or "size" of the fluctuating region or source (see ref. 21).

If the maximum cross-correlation coefficient can be expressed as

$$
R_{\max }=f\left(\frac{\zeta}{\tau}\right)=\text { const } \frac{\zeta}{\tau} \text {, }
$$

then it is also possible to define convective or rotative velocities of the fluctuating disturbance (21). Thus, in summary to describe adequately a fluctuating region or "noise" source, three types of measurements are required, namely:

1) Mean-square, $\overline{e^{2}(t)}$, or root-mean-square, $\quad \sqrt{\overline{\mathrm{e}^{2}(\mathrm{t})}}$

2) Spectral density function, $F(f)$, or amplitude vs. frequency, $A(f)$ (as in the present paper)

3) Cross-correlation function as a function of separation and time-delay between the two signals.

\section{References}

1. Meissinger, H. F. and Benson, R. A., "Design Characteristics of Solar Flectric Spacecraft for Multiple Interplanetary Missions, "AIAA Paper 70-645, San Diego, Calif., 1970.

2. Anon., "Study of a Solar Electric Multi-Mission Spacecraft", TRW-09451-6001-RO-03-Rev., Mar. 1970, TRW Systems Group, Redondo Beach, Calif.; also CR-109435, Mar. 1970, NASA.

3. Horio, S. P., "Solar Electric Propulsion Asteroid Belt Mission Study. Vol. 1: Summary Report," SD-70-21-1, Jan. 1970, North American Rockwell Corp., Downey, Calif.; also CR-109342, Jan. 1970, NASA.

4. Anon., "Thermoelectric Outer Planets Spacecraft (TOPS)," JPL-TM-33-589, Apr. 1973 , Jet Propulsion Laboratory, Pasadena, Calif.; also CR-131451, Apr. 1973, NASA.

5. Gardner, J. A., "Solar Electric Propulsion System Integration Technology (SEPSIT), Vol. 1: Technical Summary," JPL-TM-33-583, Vol. 1, Nov. 1972, Jet Propulsion Laboratory, Pasadena, Calif.; also CR-130701, Nov. 1972, INASA.

6. Serafini, John S., "Correlation Measurements of Plasma Fluctuations in a Hall-Current Accelerator," Bulietin of the American Physical Society, Vol. 13, no. 2, Feb. 1968, p. 278 .

7. Serafini, John S., "Utility of Conventional Turbulence Experimental Methods in the Study of Plasma Fluctuations, "Bulletin of the American Physical Society, Vol. 13, no. 5 , May, 1968, p. 824; also TM X-52371, 1967, NASA.

8. Serafini, John S., "Measurement of the Coherent Oscillations and Turbulence in Plasma Using Resistivity and Capacitivity Coupled Probes," TM X-1975, March, 1970, NASA.

9. Lee, H. K. and Ribner, H. S., "Direct Correlation of Noise and Flow of a Jet," Journal of the Acoustical Society of America, Vol. 52, no. 5 (Part 1), Nov. 1972, pp. 1280-1290.

10. Ion Device Physics Department Staff, "Low Voltage 30-cm Ion Thruster, Final Report," Feb. 1972, Hughes Research Laboratories, Malibu, Calif.; also CR-120919, Feb. 1972, NASA.

11. Rawlin, V. K., "Performance of $30-\mathrm{cm}$ Ion Thrusters With Dished Accelerator Grids", Proposed Paper for 10th Electric Propulsion Conference.

12. Poeschel, R. L., "The Variable Magnetic Baffle as a Control Device for Kaufman Thrusters," AIAA Paper 72-488, Bethesda, Maryland, 1972.

13. Bechtel, R. T., "A 30-cm Diameter Bombardment Thruster With a Variable Magnetic Baffle," AIAA Paper 72-489, Bethesda, Maryland, 1972. 
14. Terdan, Fred F. and Bechtel, Robert T., "Control of a 30-cm Diameter Mercury Bombardment Thruster," Proposed paper for $10^{\text {th }}$ Flectric Propulsion Conference.

15. Serafini, J. S., "Some Observations on the Relationship Between Instabilities and Turbulence In Plasma, " NASA TM X-52501, 1968.

16. Chen, F. F., "Spectrum of Low- $\beta$ Plasma Turbulence," Physical Review Letters, Vol. 15, No. 9, Aug. 1965, pp. 381-383.

17. Tchen, C. M., "A Cascade Theory of Turbulence," Bulletin of the American Physical Society, Vol. 13, No. 5, May 1968, pp. 803.
18. Wilhelm, H. E., "Theoretical Investigations on Plasma Processes in the Kaufman Thruster."

Annual Report, June 1, 1973, Dept. of Physics, Colorado State University (NASA Grant NGR06-002-147).

19. Martin, A. R., "Indications of Anomalous Diffusion in an Ion Engine Discharge," Plasma Physics, Vol. 14, No. 2, Feb. 1972, pp. 123132

20. Cohen, A. J., "Onset of Anomalous Diffusion in Electron-Bombardment Thruster, "TN D-3731, Nov. 1966, NASA.

21. Serafini, J. S., "Wall-Pressure Fluctuations and Pressure-Velocity Correlations in a Turbulent Boundary Layer," TR R-165, Dec. 1963, NASA. 


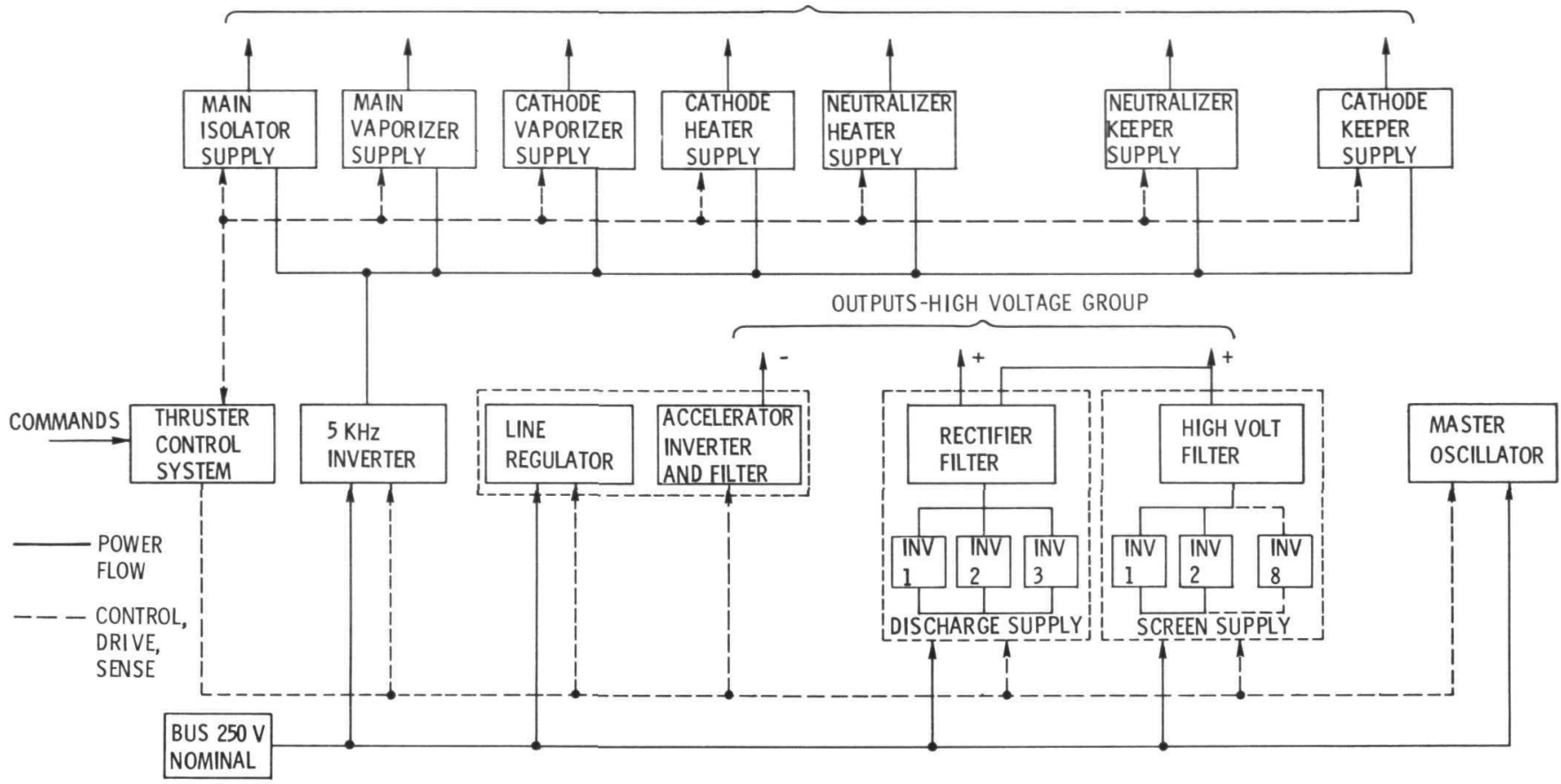

Figure $1 .-30 \mathrm{~cm}$ thruster power system block diagram.

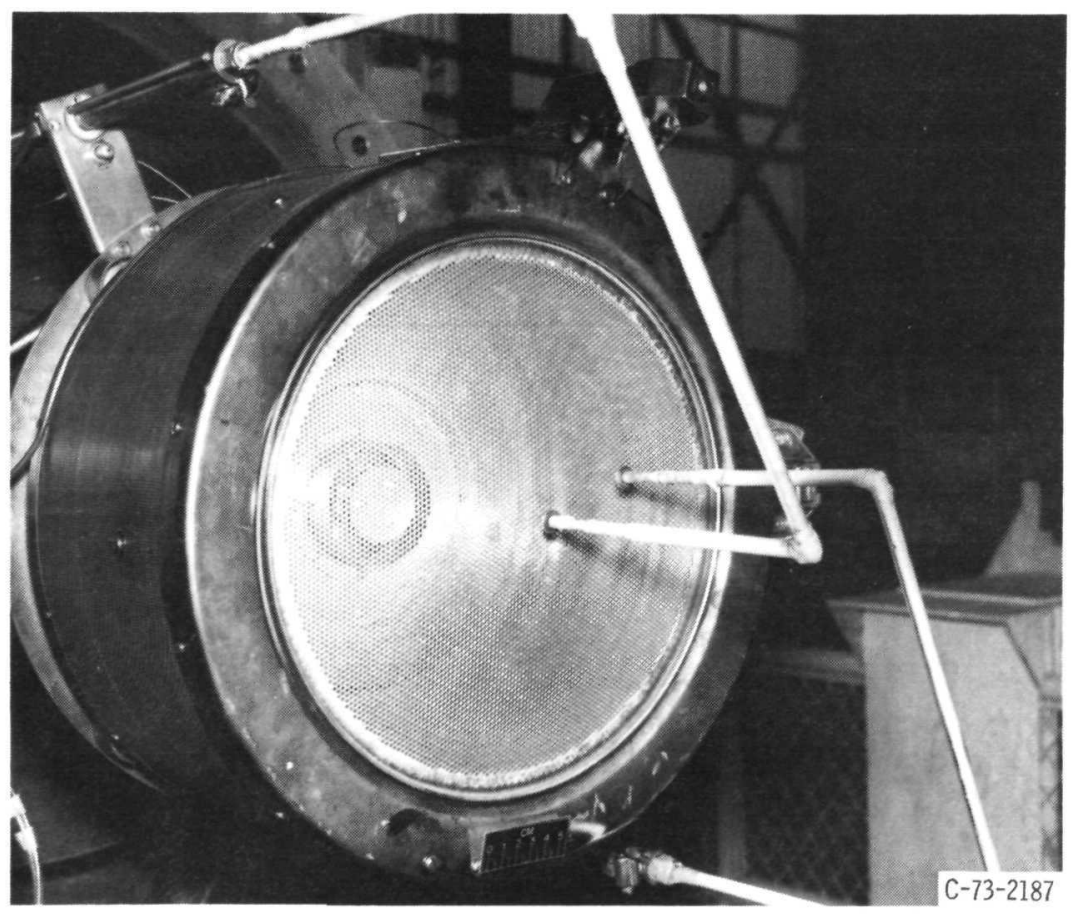

Figure 2. $-30-\mathrm{cm}$ thruster and beam probes. 


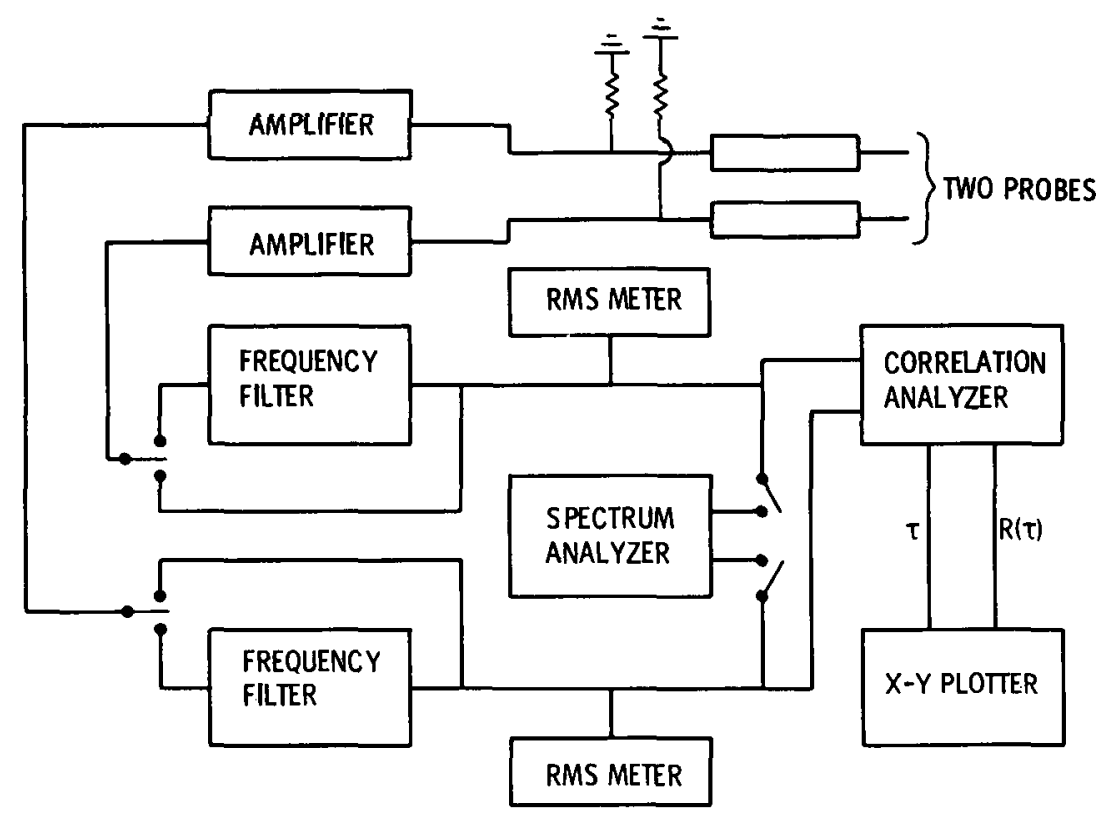

Figure 3. - Schematic diagram of instrumentation used to obtain cross-correlations and spectra for plasma fluctuations.

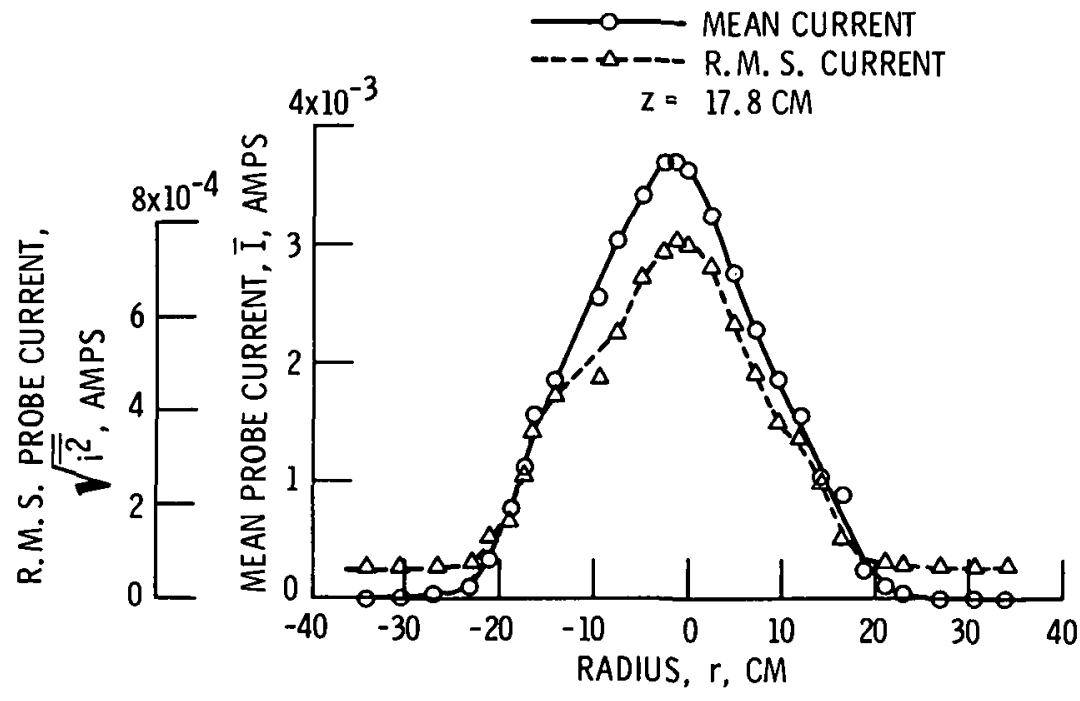

Figure 4. - Radial variation of the root-mean-square magnitude of the fluctuations and time-mean currents to the ion beam probe. $\mathrm{J}_{\mathrm{B}}=1.45 \mathrm{amp}, \mathrm{J}_{\mathrm{mb}}=6.0 \mathrm{amps}, \Delta \mathrm{V}_{\mathrm{I}}=37.4$ volts, and $\mathrm{J}_{\mathrm{e}}=7.26$ amp.

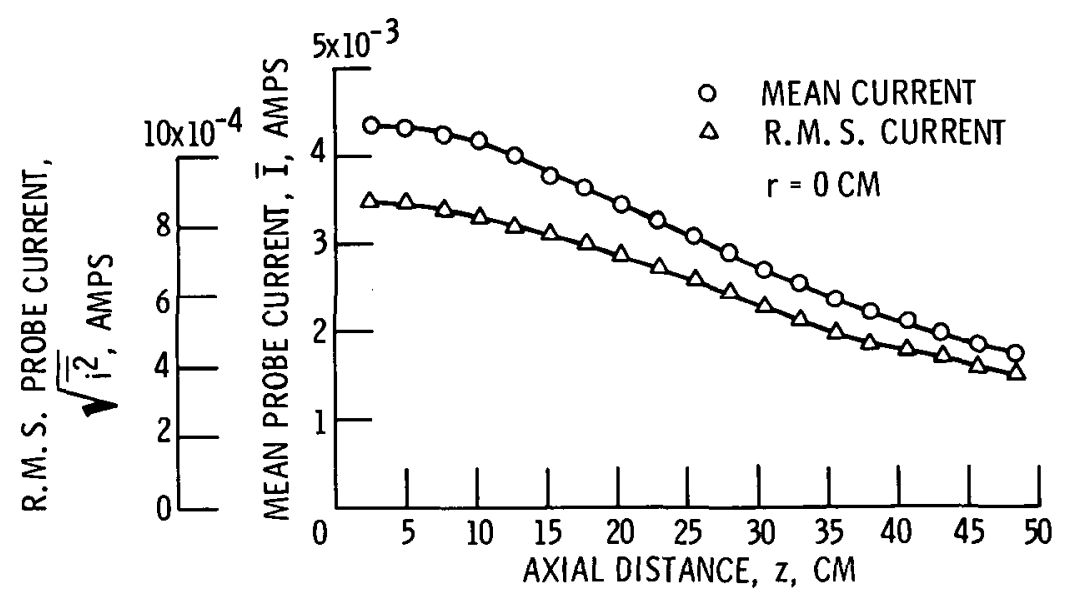

Figure 5. - Axial variation of the root-mean-square magnitude of fluctuating and time-mean currents to the ion beam probe. $J_{B}=$ $1.45 \mathrm{amp}, \mathrm{J}_{\mathrm{mb}}=6.0, \Delta \mathrm{V}_{\mathrm{I}}=37.4$ volts, and $\mathrm{J}_{\mathrm{e}}=7.26 \mathrm{amp}$. 


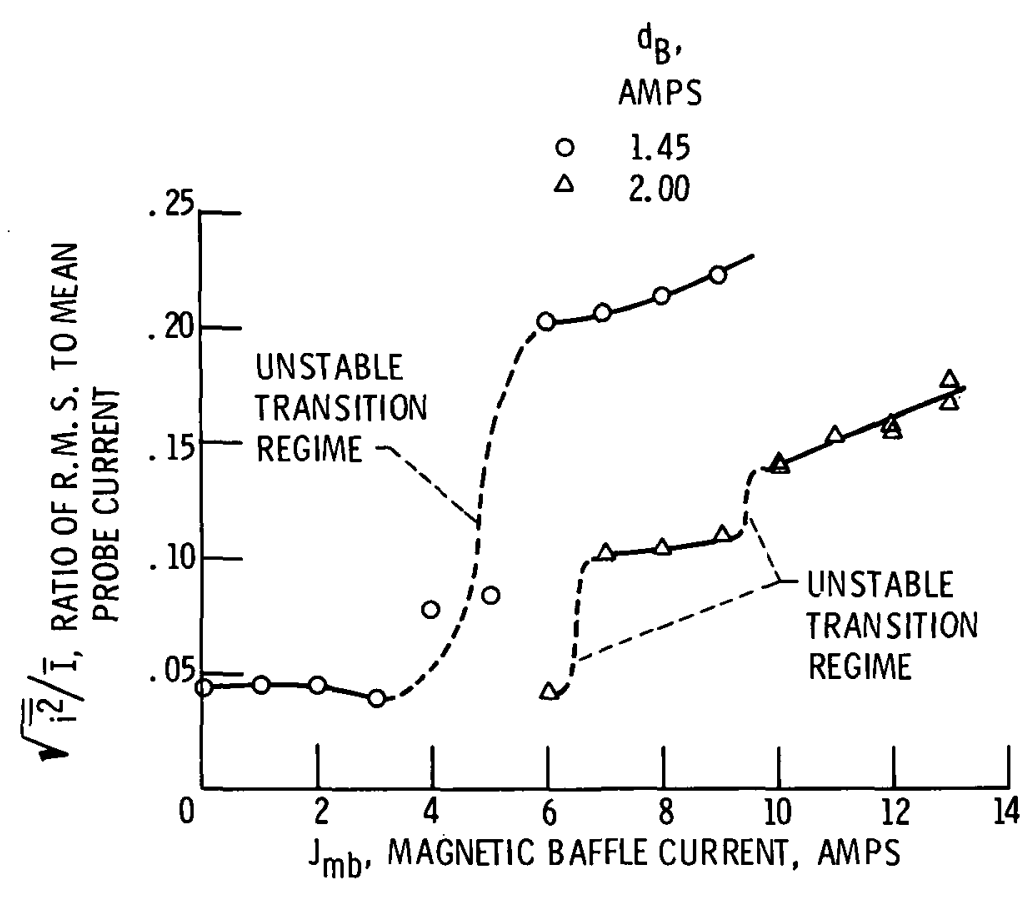

Figure 6. - Variation of the intensity (ratio of root-meansquare magnitude to time-mean) of the ion beam probe fluctuations with $\mathrm{J}_{\mathrm{mb}}$, the magnetic baffle current, $r=0, z=17.8 \mathrm{~cm}$, and $J_{B}=1.45$ and 2.00 .

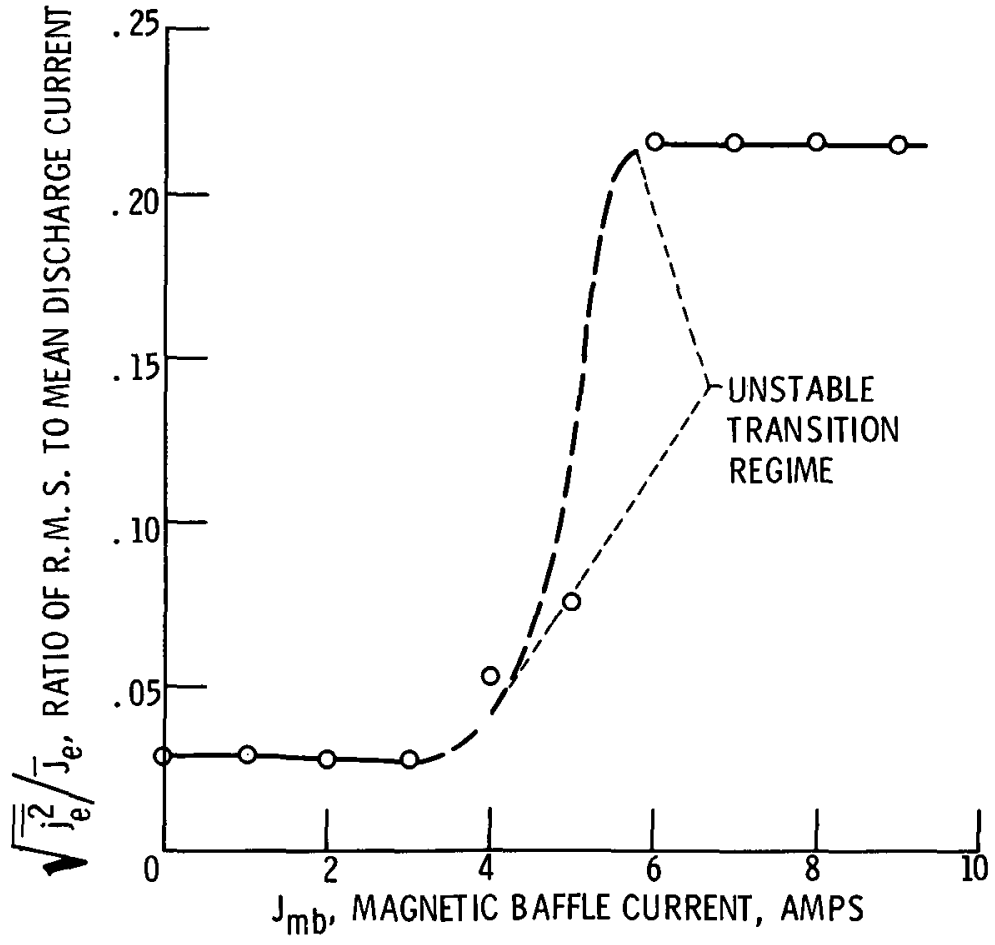

Figure 7. - Variation of the intensity (ratio of root-meansquare magnitude to time-mean) of the discharge current fluctuations with $J_{m b}$, the magnetic baffle current, $\mathrm{J}_{\mathrm{B}}=1.45$ amps and $\mathrm{J}_{e}=7.20$ amps. 


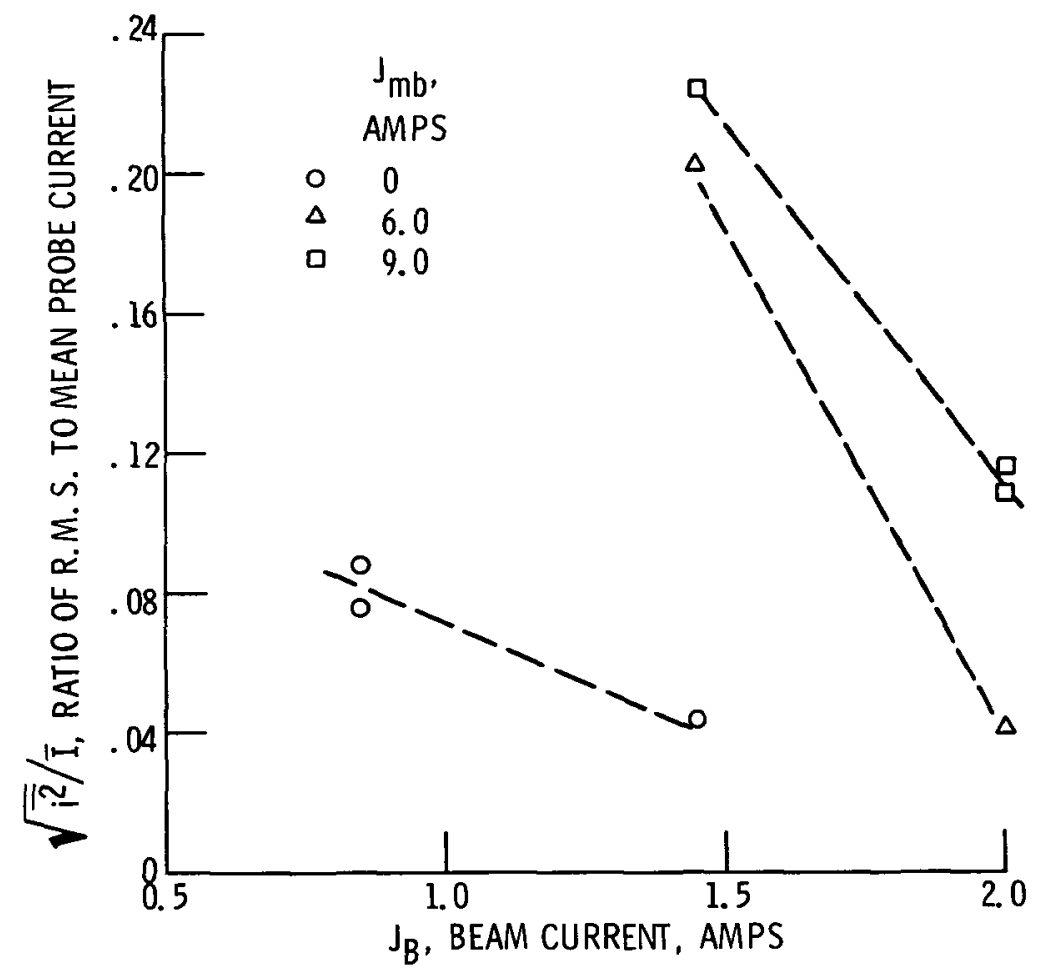

Figure 8. - Variation of the intensity (ratio of root-meansquare magnitude to time-mean) of the ion beam probe fluctuations with $J_{B}$, the beam current, for several values of $\mathrm{J}_{\mathrm{mb}}$, the magnetic baffle current.

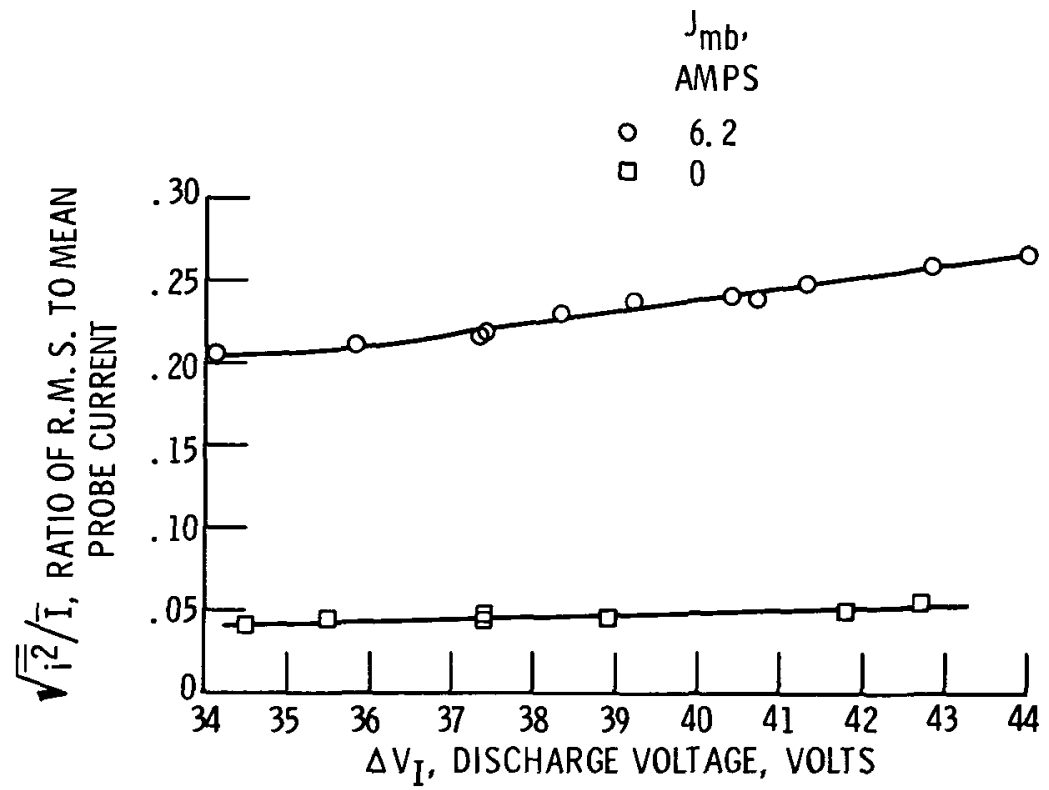

Figure 9. - Variation of the intensity (ratio of root-meansquare magnitude to time-mean) of the ion beam probe fluctuations with $\Delta V_{I}$, the discharge voltage, $J_{B}=1.45$ amps, and $J_{e}=7.26$ amps. 


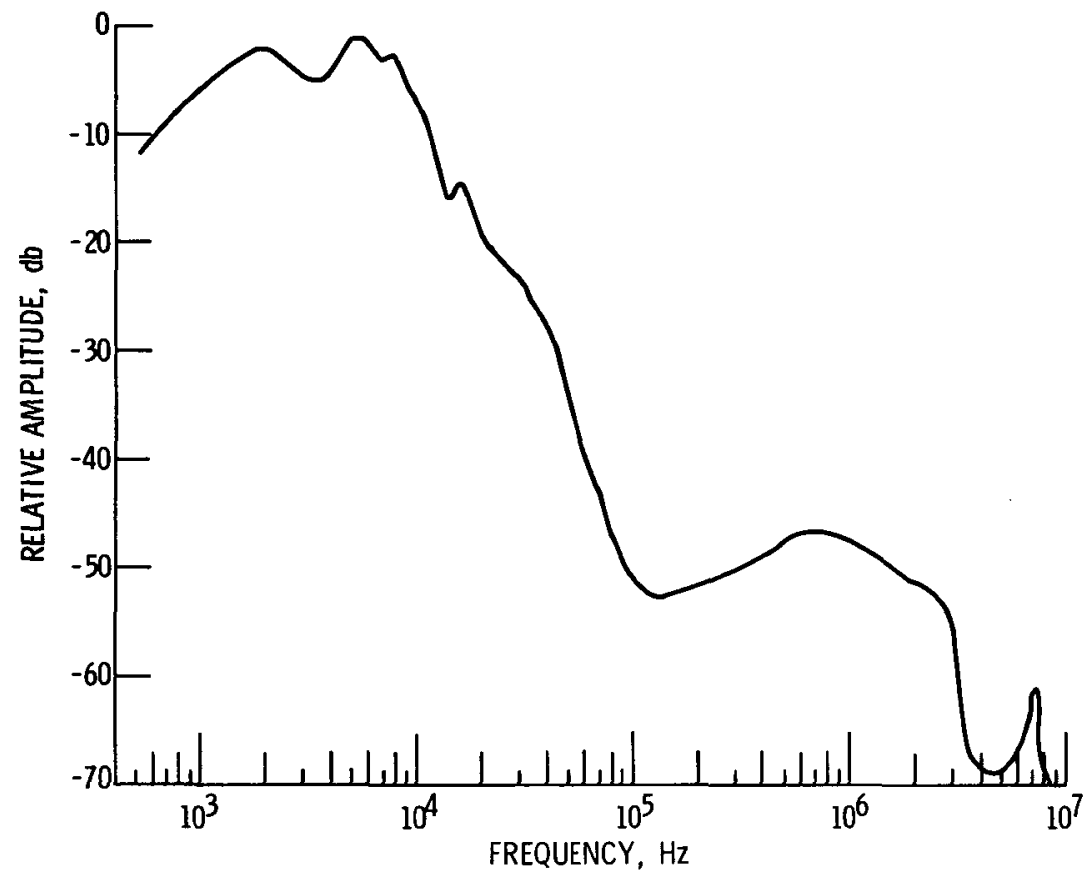

Figure 10. - Relative amplitude as a function of frequency for the ion beam probe current fluctuations, $r=0, z=17.8 \mathrm{~cm}, J_{B}=2.0$ amps, $J_{m b}=13.0$ amps, and $\vec{J}_{e}=10.0$ amps.

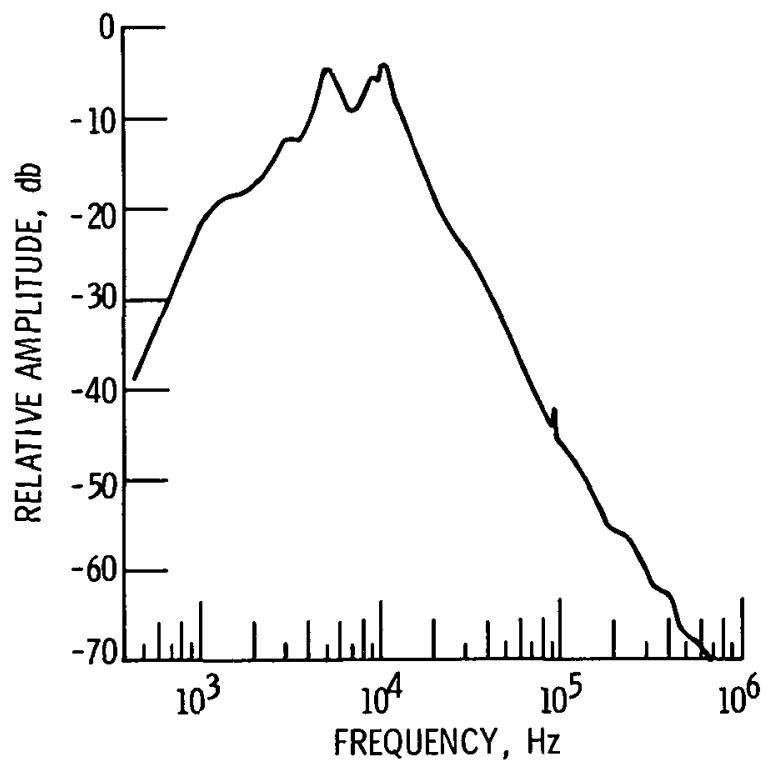

Figure 11. - Relative amplitude as a function of frequency for the discharge current fluctuations, $\bar{J}_{\mathrm{e}}=10.0$ amps, $\mathrm{J}_{\mathrm{mb}}=13.0$ amps $\left(J_{B}=2.0\right.$ amps $)$. 


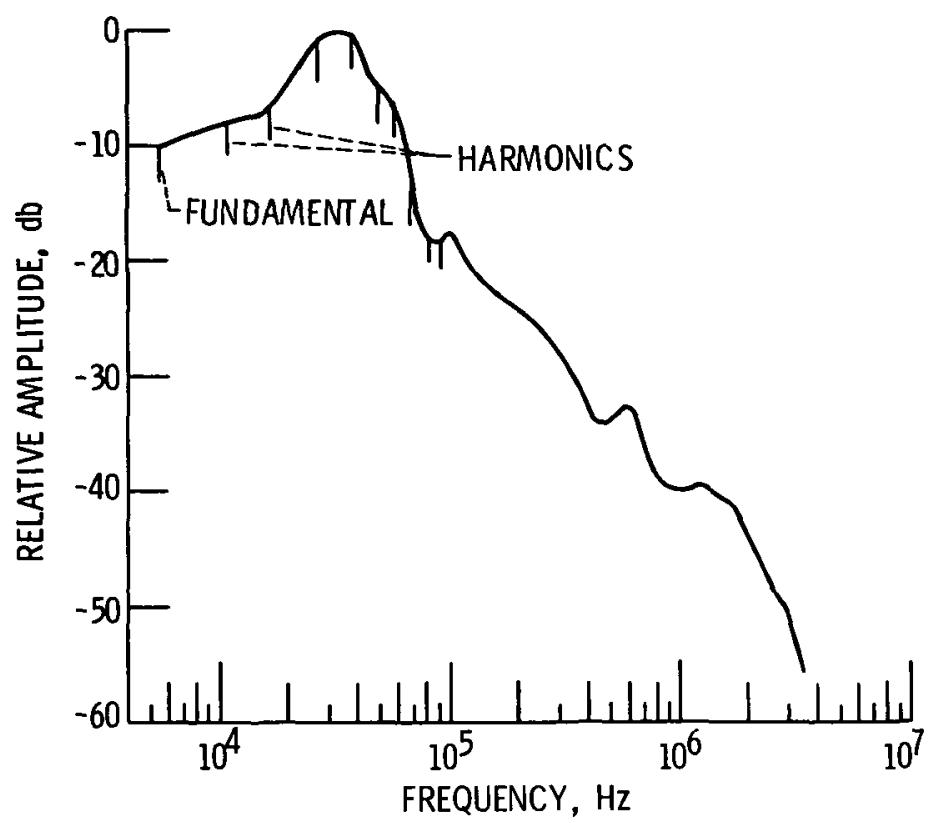

Figure 12. - Relative amplitude as a function of frequency for the neutralizer keeper current fluctuations, $\bar{J}_{n k}=$ $200 \mathrm{amps}\left(\mathrm{J}_{\mathrm{B}}=20 \mathrm{amps}, \mathrm{J}_{\mathrm{mb}}=13.0 \mathrm{amps}\right) \sqrt{\mathrm{j}_{\mathrm{nk}}^{2}}=0.29$ amps.

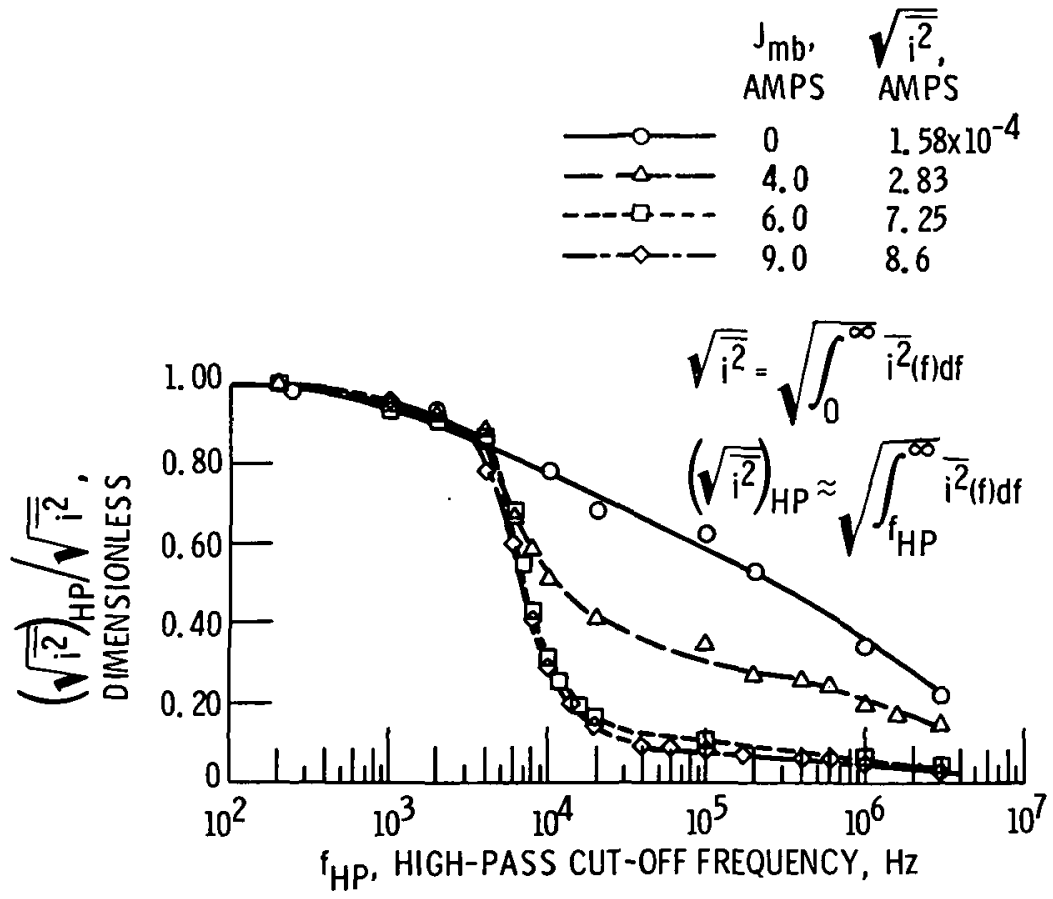

Figure 13. - Variation of the high-pass band of the r.m.s. magnitudes of the ion beam probe fluctuations as a function of the cut-off frequency, $\mathfrak{f}_{\mathrm{HP}}$, of the high-pass band for several magnetic baffle currents, $J_{m b}\left(J_{B}=1.45\right.$ amps $)$. 


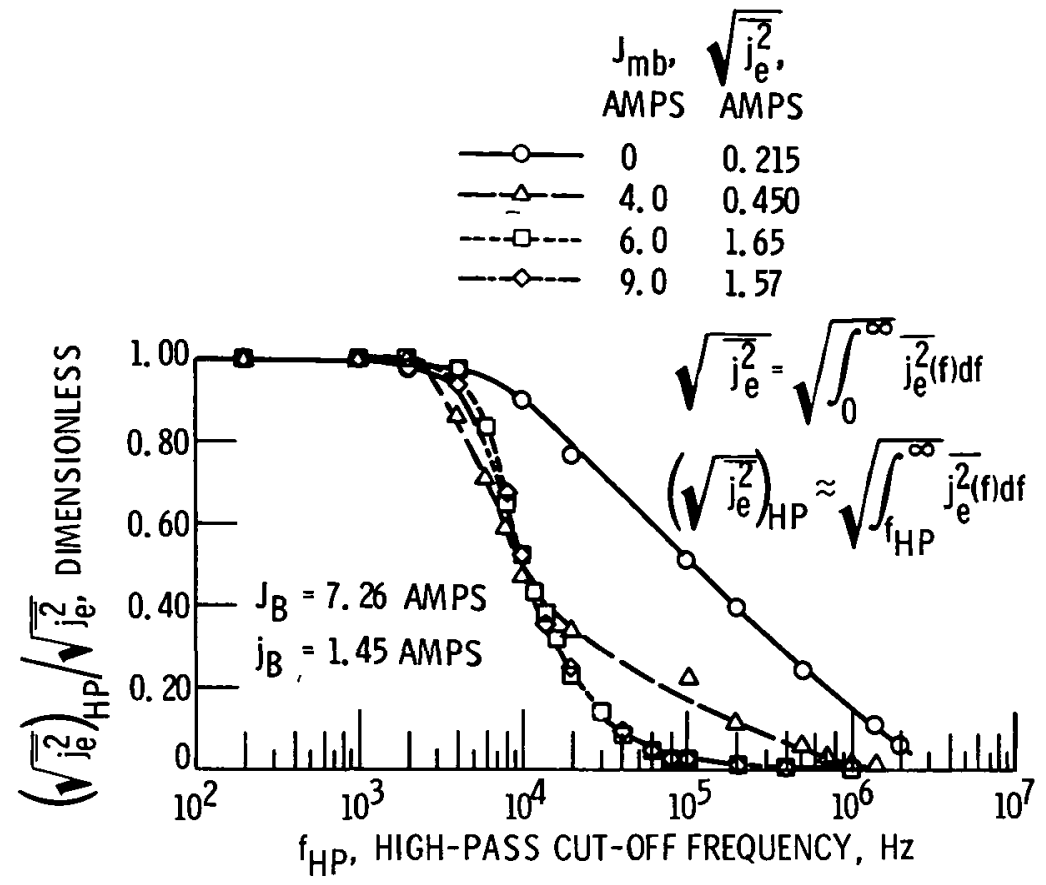

Figure 14. - Variation of the high-pass band of the r.m.s. magnitudes of the discharge current fluctuations as a function of the cut-off frequency, $f_{H P}$, of the high-pass band for several magnetic baffle currents, $\mathrm{J}_{\mathrm{mb}}\left(\mathrm{J}_{\mathrm{B}}=\right.$ 1.45 amps).

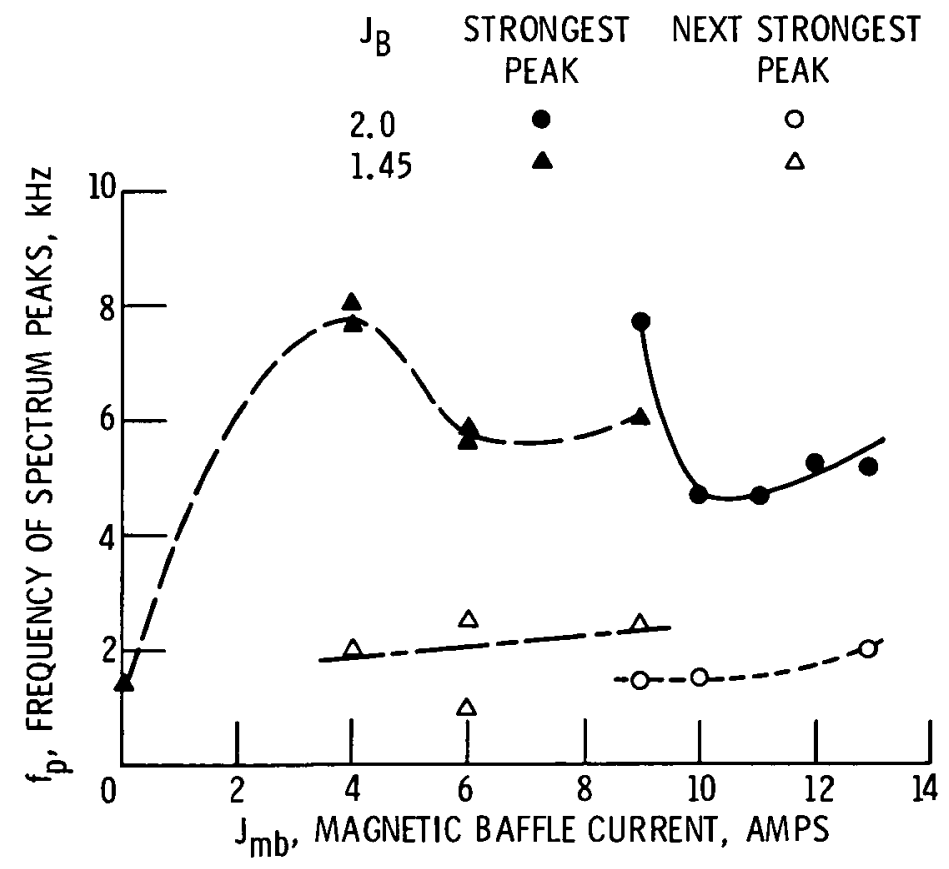

Figure 15. - Variation of the peak-frequencies of the ion beam fluctuations as a function of $\mathrm{J} \mathrm{mb}$, the magnetic baffle current (for $J_{B}=1.45$ and 2.00). 


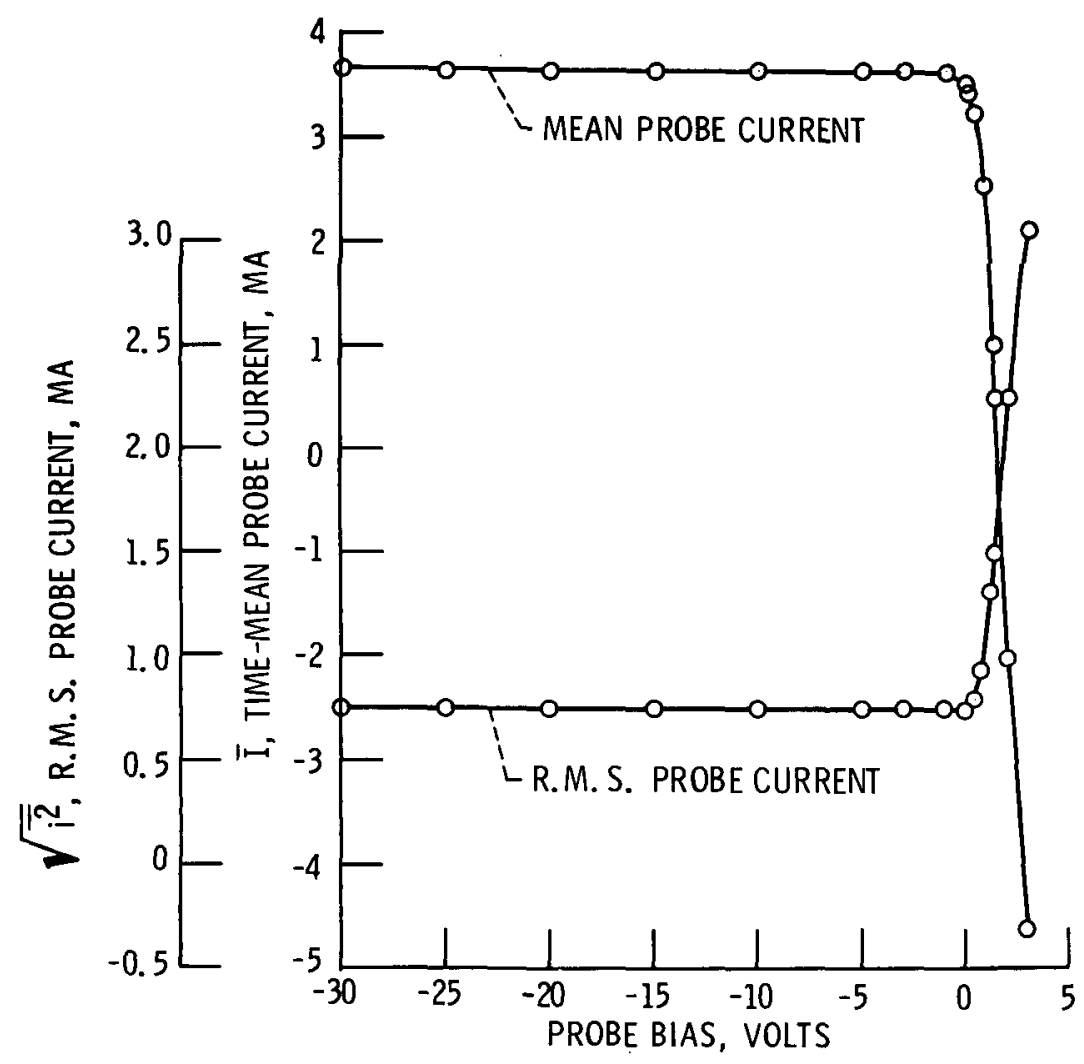

Figure 16. - Variation of r.m. s. magnitude of the fluctuating and time-mean ion beam probe currents as a function of probe bias voltage $\left(J_{B}=1.45\right.$ amps, $J_{m b}=6.0$ amps, $r=0$, and $z=$ $17.8 \mathrm{~cm})$.

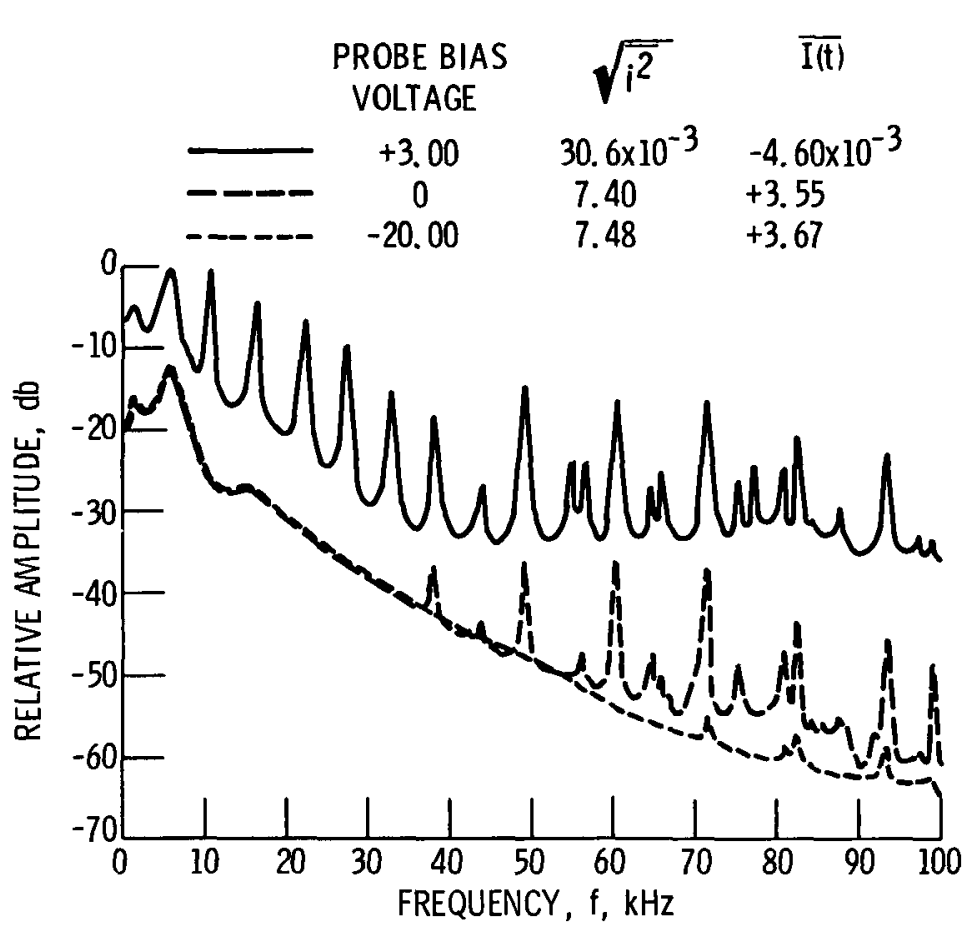

Figure 17. - Variation of relative amplitude in $\mathrm{db}$ for the ion beam probe fluctuations as a function of frequency for three probe bias voltages $\left(r=0, z=17.8 \mathrm{~cm}, J_{B}=\right.$ 1.45 and $\mathrm{J}_{\mathrm{mb}}=6.0$ amps). 


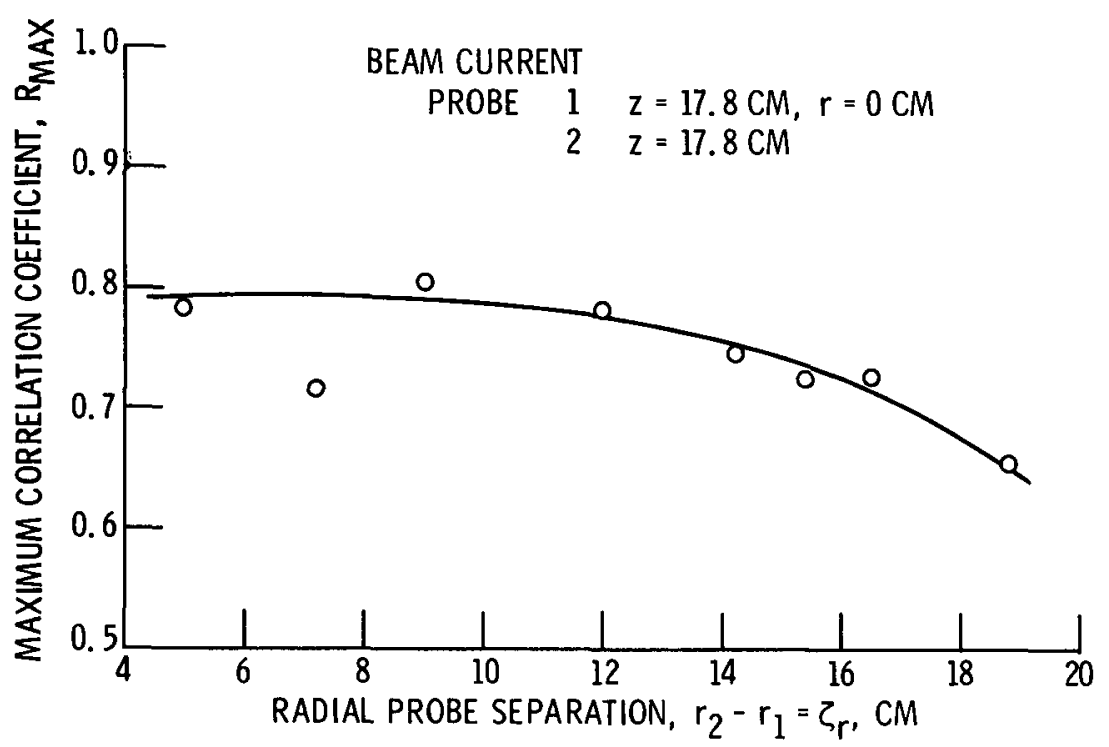

Figure 18. - Variation of $R_{\max }$ of the beam fluctuations as a function of the radial probe separation with $J_{B}=1.45$ amps and $J_{m b}=$ 6.0 amps.

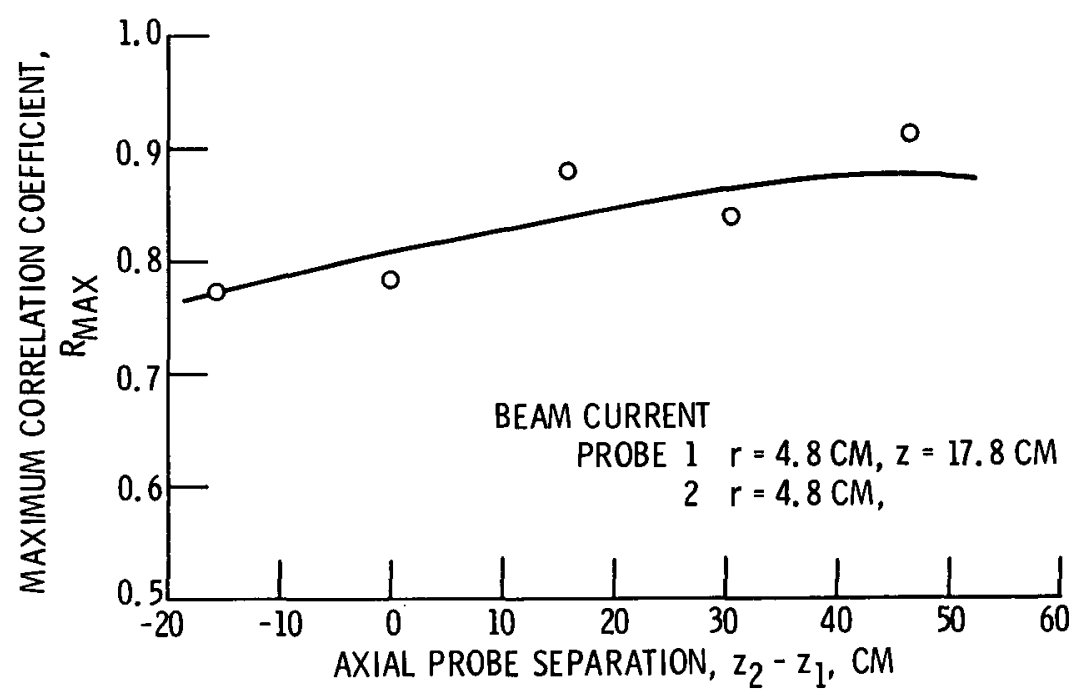

Figure 19. - Variation of $R_{\max }$ of the beam fluctuations as a function of the axial probe separation with $J_{B}=1.45$ amps and $J_{m b}=6.0$ amps.

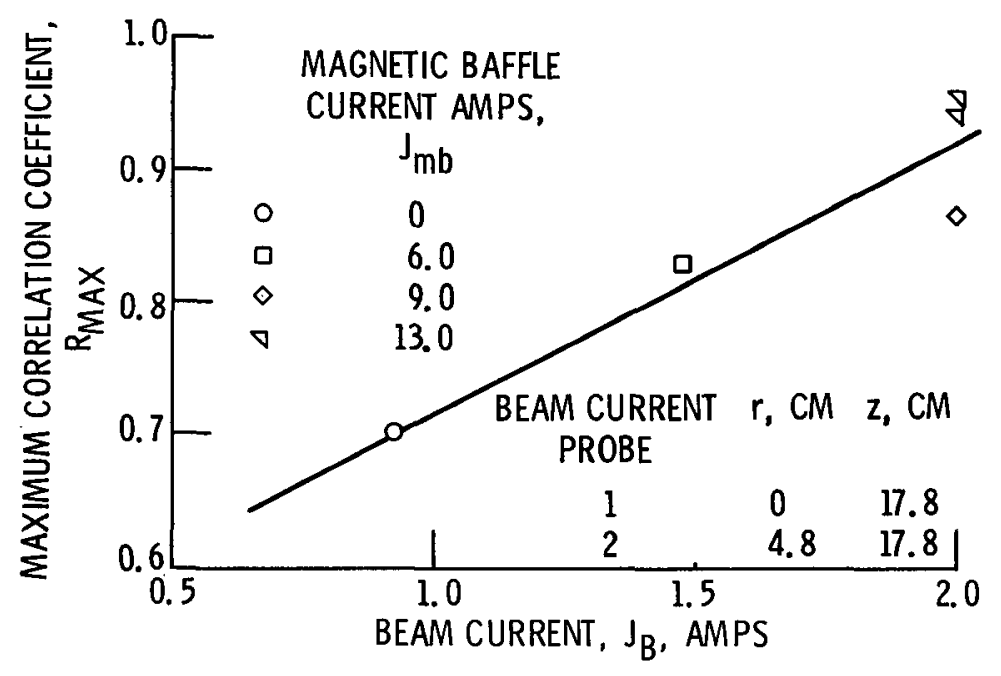

Figure 20. - Variation of $R_{\max }$ of the beam fluctuations as a function of beam current, $J_{B}$, for various magnetic baffle currents, $\mathrm{J}_{\mathrm{mb}}$. 


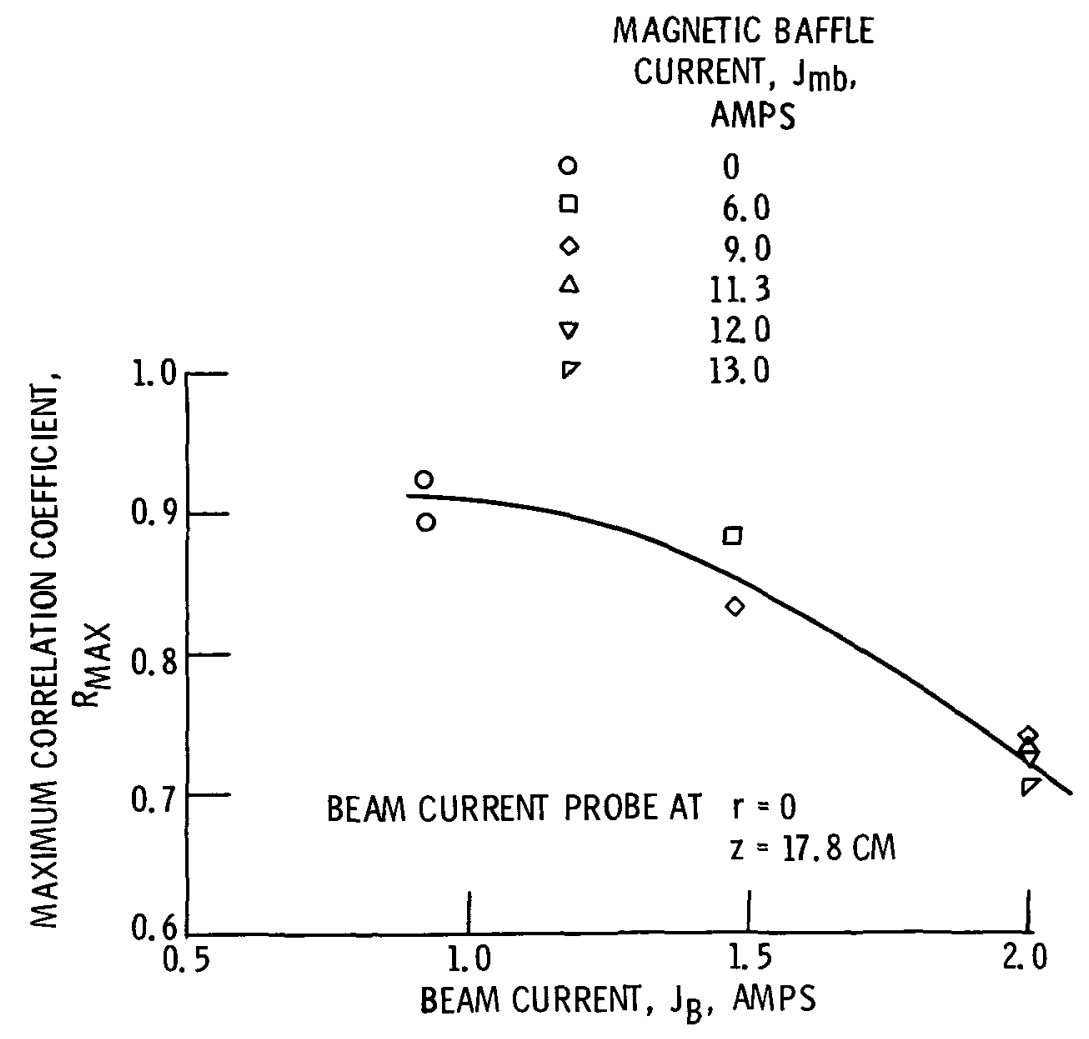

Figure 21. - Variation of $R_{\max }$ of the cor relations of the beam and discharge current fluctuations as a function of the beam current, $J_{B}$, for various magnetic baffle currents.

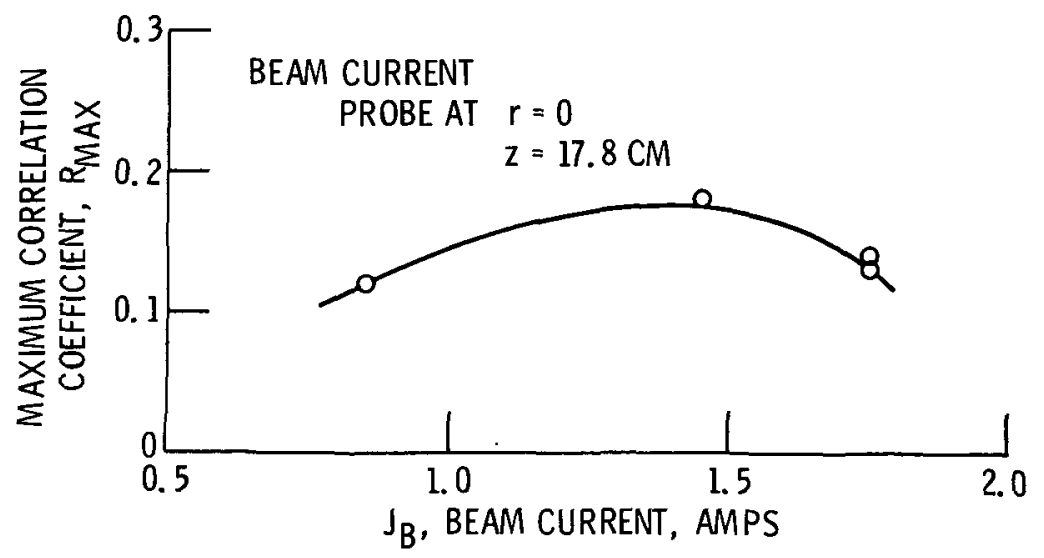

Figure 22. - Variation of $R_{\max }$ of the correlations of the beam and neutralizer keeper current fluctuations as a function of the beam current, $\mathrm{J}_{\mathrm{B}}$. 